\title{
Novel Air-filled Waveguide Transmission line based on Multi-layer Thin Metal Plates
}

\author{
Abbas Vosoogh, Herbert Zirath, Fellow, IEEE, and Zhongxia Simon He, Senior Member, IEEE
}

\begin{abstract}
This paper presents a novel way of constructing wave guiding structures by stacking several thin metal plates for millimeter-wave applications. The metallic layers do not require any electrical contacts among them. An air-filled multilayer waveguide (MLW) transmission line is successfully designed and manufactured at D-band. Five vertically stacked thin metal layers are used to form an air-filled rectangular waveguide line. The layers are simply assembled by allowing a small air gap among them, without the need of using advanced manufacturing methods such as adhesive bonding techniques. The possible field leakage due to the air gaps is prevented by using an electromagnetic band gap (EBG) structure, consisting of glidesymmetric holes. A straight MLW line and a line with a double $90^{\circ}$ bends are fabricated by using chemical metal etching, as a proof of concept. The measurement results of the straight line show that the reflection coefficient is better than -18 dB with average insertion loss of $0.02 \mathrm{~dB} / \mathrm{mm}$ over the frequency band 110-170 GHz. For the double $90^{\circ}$ bends line, the reflection coefficient is better than $-18 \mathrm{~dB}$ with an average insertion loss value of $0.02 \mathrm{~dB} / \mathrm{mm}$ over the frequency band 110-150 GHz. The proposed concept could be an excellent candidate for designing compact and thin passive waveguide components and active components packaging, with a great potential for low-cost, light weight and mass-producible at millimeter-wave frequencies.
\end{abstract}

Index Terms-Electromagnetic band gap (EBG), multi-layer waveguide (MLW), chemical metal etching, millimeter-wave, waveguide.

\section{INTRODUCTION}

$\mathbf{T}$ RANSMISSION lines are the fundamental building blocks of any high frequency systems to guide, manipulate and transmit signals. They play an important role in passive and active components design and integration. Conventional guiding structures, such as metal-pipe hollow waveguides, planar transmission lines (e.g., microstrip, Substrate Integrated Waveguide (SIW), and coplanar waveguide) are popular and widely used technologies. Hollow waveguides show superior performance in terms of loss, whilst the planar technologies exhibit compact and flexible manufacturing advantages.

Due to the increasing demand for higher data rate transmission and the existing overcrowded frequency spectrum, the millimeter-wave frequency band $(30-300 \mathrm{GHz})$ has received increasing attention to provide more available bandwidth and higher capacity [1], [2]. Using higher frequencies has the advantage of reducing the size of components, e.g., high gain

A. Vosoogh is with the Electrical Engineering Department at Chalmers University of Technology, Gothenburg, Sweden (e-mails: abbas.vosoogh@chalmers.se).

Z. S. He and H. Zirath are with the Department of Microtechnology and Nanoscience at Chalmers University of Technology, Gothenburg, Sweden. antennas with small aperture sizes, which led to more compact systems. However, increasing the operating frequency leads to enormous challenges in fabrication of components with small features. Mechanically small features in 3-D hollow waveguide structures requires precise and costly manufacturing techniques at high frequencies, while the high loss of planar transmission lines limits their usage, especially in electrically big components such as array antennas. For example, high-gain array antennas with physically small size have been presented at $120 \mathrm{GHz}$ [3] and $350 \mathrm{GHz}$ [4] based on rectangular hollow waveguide with a complex and highcost manufacturing technique, where they have etched especial patterns in several thin metal plates and bonded them under high pressure and temperature condition in vacuum.

Substrate based planar transmission line technologies offer the advantages of easy fabrication and integration. However, they show higher losses mainly due to the presence of dielectric and field leakage. New substrate materials, such as Liquid Crystal Polymer (LCP), have been used for high frequency applications to have a low loss tangent and a stable dielectric constant. In [5] the electrical properties of LCP substrate has been characterized at D-band. It has been shown that the insertion loss of a microstrip line in 2 mil substrate increases from $0.176 \mathrm{~dB} / \mathrm{mm}$ to $0.33 \mathrm{~dB} / \mathrm{mm}$ over the frequency band 110-170 GHz. Surface roughness becomes more important at frequencies above $100 \mathrm{GHz}$, and has a significant impact on the total system loss [6]. The performance of a SIW transmission line and the impact of surface roughness on dissipative loss has been study at D-band in [6]. The measured results show that the unpolished prototypes exhibit insertion loss around $0.8 \mathrm{~dB} / \mathrm{mm}$ and by polishing the substrate, an average insertion loss of $0.35 \mathrm{~dB} / \mathrm{mm}$ has been achieved.

Metallic air-filled hollow waveguides are attractive low loss solutions in spite of the fact that fabrication of complex 3D waveguide structures is challenging at high frequencies. Computerized Numerically Controlled (CNC) milling and diesink Electric Discharge Machining (EDM) are conventional manufacturing techniques to fabricate waveguide structures in low volume. The waveguide structures with EDM and CNC machining are usually fabricated in E-plane split-blocks in order to have a simple assembly and avoid field leakage. In [7] rectangular waveguide lines in different planes cuts and different material (e.g., aluminum and brass) have been fabricated by CNC milling. The lowest insertion loss has been reported for the E-plane split-block prototype in aluminum with a loss of around $0.005 \mathrm{~dB} / \mathrm{mm}$ over the frequency band $75-110 \mathrm{GHz}$. Alternative fabrication approaches with features of low-cost, suitable for mass production and easy integration have been 
studied and developed recently, especially for millimeter-wave applications.

Adaptive manufacturing (3-D printing) [8], [9], micromachining technology [10], [11], and micro-molding [12] have been recently introduced as alternative manufacturing techniques for high frequency applications. The 3-D printing technology has advantages of low cost and rapid prototyping, even for fabrication of complex structures. In [13] high-resolution metal-coated stereolithography apparatus (SLA) technique is used to manufacture a rectangular waveguide and a bandpass filter in a E-plane split-block configuration at W-band. It has been demonstrated a measured average dissipative loss of $0.013 \mathrm{~dB} / \mathrm{mm}$ for the copper coated waveguide line at $\mathrm{W}$ band. A $25 \mathrm{~mm}$ long straight copper-plated plastic waveguide is printed in [8] with the same technique (SLA) at H-band (220 - $325 \mathrm{GHz}$ ) showing an insertion loss of $0.014 \mathrm{~dB} / \mathrm{mm}$. Waveguide structures can be also manufactured in one piece by using direct metal printing. In [9], rectangular waveguide lines by using selective laser melting (SLM) technique are fabricated by melting $\mathrm{Cu}-15 \mathrm{Sn}$ powder. The insertion losses of $0.075 \mathrm{~dB} / \mathrm{mm}, 0.019 \mathrm{~dB} / \mathrm{mm}$, and $0.12 \mathrm{~dB} / \mathrm{mm}$ have been reported for waveguide lines at E-, D-, and H-band, respectively.

Micromachining is a high-precision elaborate manufacturing method to make high-performance waveguide devices. A micromachined gold-plated H-plane split-block rectangular waveguide with a very low insertion loss of 0.008-0.016 $\mathrm{dB} / \mathrm{mm}$ at D-band is reported in [14]. Moreover, a siliconbased micromachined bandpass rectangular waveguide filter is presented at D-band in [10], with insertion losses better than $0.5 \mathrm{~dB}$ and $0.7 \mathrm{~dB}$ for third and fifth order filters, respectively. Silicon micromachining is limited by maximum etching depth and it is more suitable for higher frequencies, e.g., above $250 \mathrm{GHz}$, since the physical dimensions are relatively large at W- and D-band for this technique. In [11], the use of SU-8 photoresist micromachining technology to manufacture rectangular waveguide lines in D- and H-band are studied by using 5 silver plated layers. An insertion loss of better than $0.03 \mathrm{~dB} / \mathrm{mm}$ is reported in both bands. A W-band gold-plated plastic rectangular waveguide by using micro-molding (micro hot embossing) has been presented in [12]. It has been reported a return loss better than $14 \mathrm{~dB}$ with an insertion loss of around $0.03 \mathrm{~dB} / \mathrm{mm}$ at W-band.

Low-loss waveguide components, interconnections, and packaging solutions with futures of low cost and suitable for mass production are needed for frequencies above $100 \mathrm{GHz}$. In this paper we introduce a novel and flexible approach to make affordable air-filled waveguide structures for millimeter-wave applications. A rectangular multi-layer waveguide (MLW) line is created by stacking thin unconnected petal plates. The layers do not need to have electrical and galvanic contacts. This introduces a cost-effective manufacturing with a simple mechanical assembly, especially at high frequencies. In Section II we present the design methodology of the MLW transmission line, including the design and characterization of the electromagnetic band gap (EBG) unit cells, straight line, and a line with two $90^{\circ}$ bends. In Section III experimental results of the fabricated prototypes are presented. Finally,

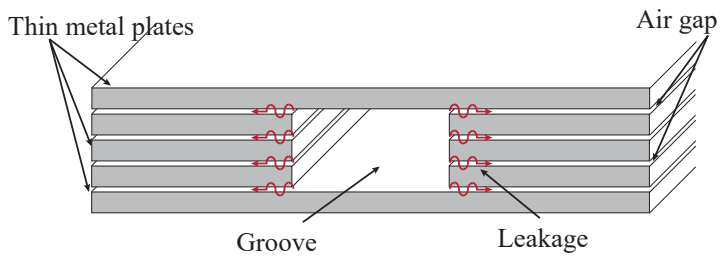

(a)

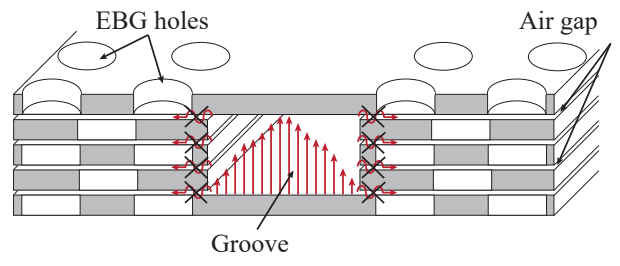

(b)

Fig. 1. Configuration of the proposed MLW transmission line. (a) Crosssection view of the WR- 6 waveguide which consists of 5 layers with small gaps between them. (b) Field-leakage suppression by using glide-symmetric holes.

Section IV gives some concluding remarks.

\section{Multi-layer Waveguide Transmission Line CONFIGURATION AND DESIGN}

The use of EBG structures and high impedance surfaces in order to prevent field leakage in metallic waveguide structures for high frequency applications was proposed by means of gap waveguide technology in [15], [16] for the first time. In this novel transmission line a low-loss waveguide was built in two metal blocks, without the need for electrical contact between them. Therefore, this waveguide structure can be manufactured with cost-effective fabrication methods, such as plastic and metal injection molding. A periodic EBG pin texture, which provides a stop-band for the desired frequency band, is used to eliminate unwanted leakages. Several waveguide components such as planar array antennas [17]-[20], filters [21], [22], diplexer [23], and integrated modules [24], [25] have been reported, which show the advantages of this technology at millimeter-wave frequencies.

A similar approach is used in [26], [27] in order to construct metallic waveguides in split-blocks without having galvanic contact. A rectangular Waveguide in H-plane split-block with holey glide-symmetric EBG structures in both plates is designed to have a simple mechanical assembly and suppressing field leakage. The two metal blocks allow to have a small gap between them and any leakage is avoided by the provided stopband from the periodic glide-symmetric holes. A waveguide phase shifter [28] and a contactless flange [29] have also designed with a similar approach.

Fig. 1 shows the configuration and arrangement of the proposed MLW transmission line. A rectangular waveguide is formed by vertically stacked unconnected thin metal plates. In Fig. 1(a), five layers are used to build up a rectangular shaped waveguide, by removing the channel in the middle layers. The height of the waveguide channel depends on the thickness and the number of the layers. Since the metal plates do not have electrical and galvanic contact, a strong field leakage occurs 


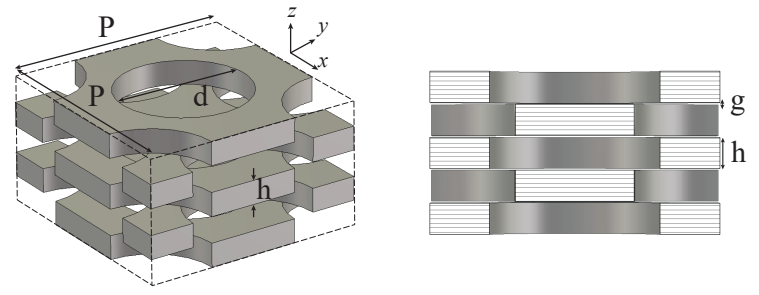

(a)
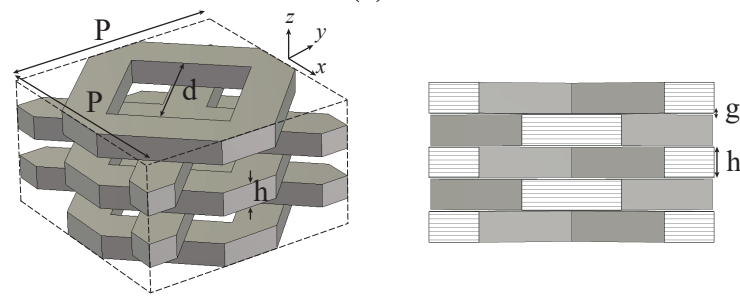

(b)

Fig. 2. Configuration of the unit cell, consisting of five metallic layers. (a) Perspective view and cross-section view of th unit cell with circular holes, (b) Perspective view and cross-section view of th unit cell with diamond-shaped holes. $(P=1.86 \mathrm{~mm}, d=1 \mathrm{~mm}, h=0.2 \mathrm{~mm}$, and $g=0.01 \mathrm{~mm})$

between the layers. In order to solve this problem we used an EBG structure to avoid any possible leakage. A periodic pattern of holes in a glide-symmetric arrangement is applied for this purpose, as shown in Fig. 1(b). The periodic holes provide stop-band for a certain frequency band which suppresses the propagation of any wave in undesired directions. This introduces a huge mechanical assembly advantage and manufacturing flexibility. Each layer can be fabricated with its special pattern separately and simply assemble afterwards without the need of a complex and costly method.

\section{A. Unit Cell Design}

The first step is to design a periodic unit cell that provides a sufficient stop-band that covers the desired operating frequency band. The geometries of the proposed unit cells are shown in Fig. 2. The unit cells consist of holes in thin metal plates. The holes are allocated in a glide-symmetric configuration. The glide symmetry is a higher order symmetry than the simple periodic pin structure in gap waveguide technology in [16]. The holes in each layers have an offset of half of the period $(\mathrm{P})$ with respect to the ones at the top and bottom layers. As shown in Fig. 2, there is a small gap ( $\mathrm{g}$ ) between the layers. The holes can have any arbitrary geometrical shape. Fig. 2 shows two unit cells with circular and diamond-shaped holes.

The dispersion diagram of the unit cells for the given values are presented in Fig. 3. We used the CST Eigenmode solver in order to calculate the dispersion diagrams. Both unit cells provide a stop-band which covers the desired frequency bands (100-200 GHz). Fig. 3 shows that, even though the unit cells consist of several unconnected layers, the structure does not support any modes within the stop-band.

Furthermore, we have also studied the effect of the unit cell parameters on the stop-band performance. Fig. 4 shows the effect of the dimension of the holes for different periodicities. The other parameters are kept the same as the unit cells of

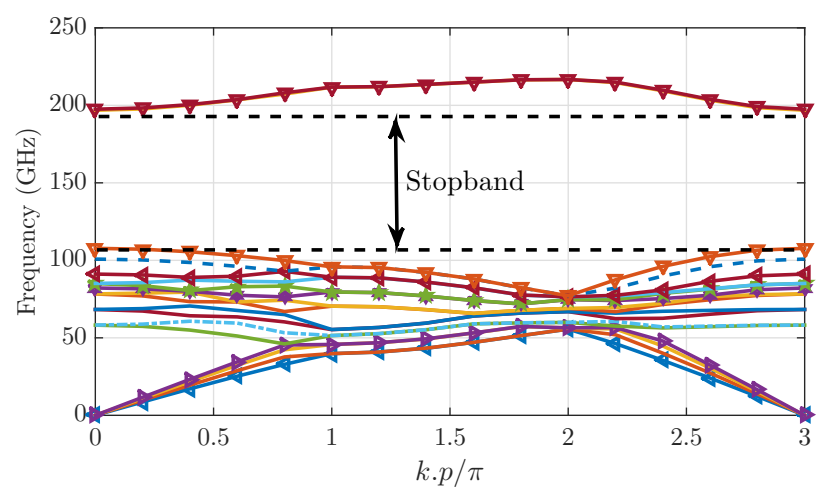

(a)

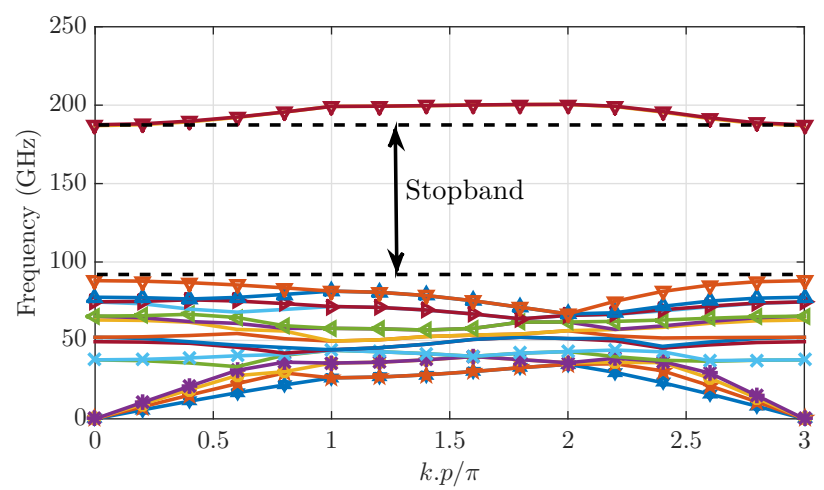

(b)

Fig. 3. Dispersion diagram for the infinite periodic unit cell with (a) circular holes, and (b) diamond-shaped holes. ( $P=1.86 \mathrm{~mm}, d=1 \mathrm{~mm}, h=0.2$ $\mathrm{mm}$, and $g=0.01 \mathrm{~mm}$ )

Fig. 2, as a reference. The lower and upper limits in the Fig. 4 are corresponding to the stop-band's start and end frequencies, respectively. Fig. 4 shows that the center frequency of the stop-band is controlled by the periodicity $(\mathrm{P})$ of the unit cell. Moreover, a maximum relative bandwidth is achieved by a certain value of the holes dimensions $(d / P)$. As can be seen, the shape of the holes has a significant impact on the relative bandwidth behavior of the stop-band.

Fig. 5 shows the relative bandwidth as a function of $d / P$ for different periodicities. For the circular hole unit cell, there is maximum bandwidth of $60 \%$ for $\mathrm{d} / \mathrm{P}$ equal to 0.52 . That means there is an optimum case when the diameter of the holes are around half of the periodicity. On the other hand, the diamondshaped hole unit cell shows a more robust performance. A maximum of more than $85 \%$ relative bandwidth is achieved for $\mathrm{d} / \mathrm{P}$ equal to 0.46 . Moreover, this unit cell show a wider bandwidth for a wider range of values of hole dimensions. This makes the unit cell with diamond-shaped holes less sensitive to manufacturing tolerances, which is very critical for frequencies above $100 \mathrm{GHz}$.

The impact of the gap between the layers is presented in Fig. 6. The other parameters have the same values as the ones presented in Fig. 2. By increasing the gap between the layers the stop-band shrinks. The unit cells do not provide a stop-band if the gap between the layers becomes bigger than a certain value. The diamond-shaped hole unit cell shows 


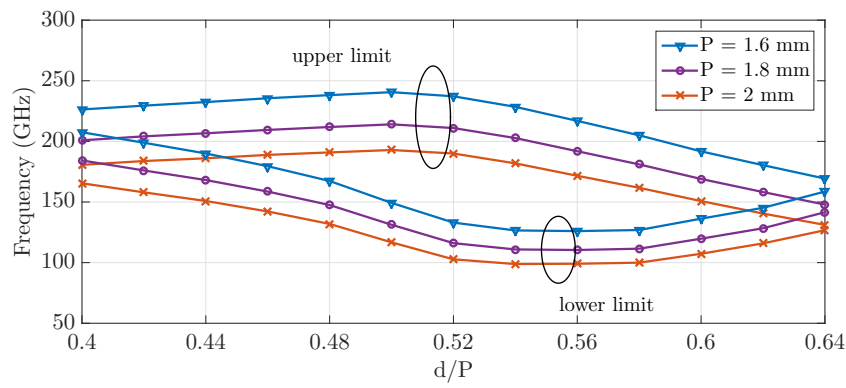

(a)

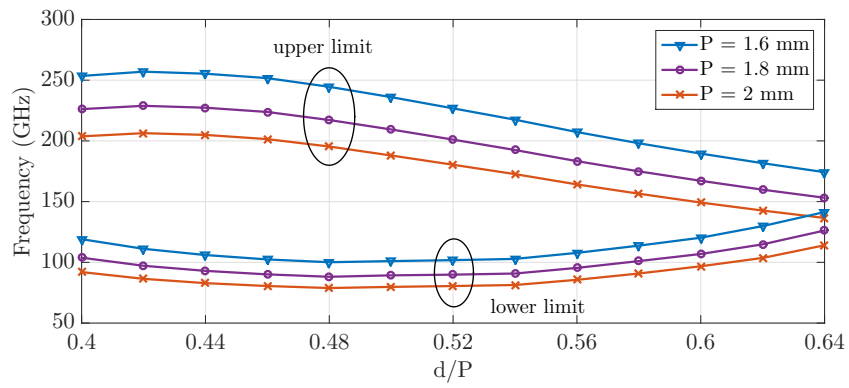

(b)

Fig. 4. Effect of diameter versus period ratio $(\mathrm{d} / \mathrm{P})$ in the stopband for a number of periodicities $\mathrm{P}$ with $\mathrm{h}=0.2 \mathrm{~mm}$ and $\mathrm{g}=0.01 \mathrm{~mm}$. (a) Unit cell with circular holes, (b) Unit cell with diamond-shaped holes.

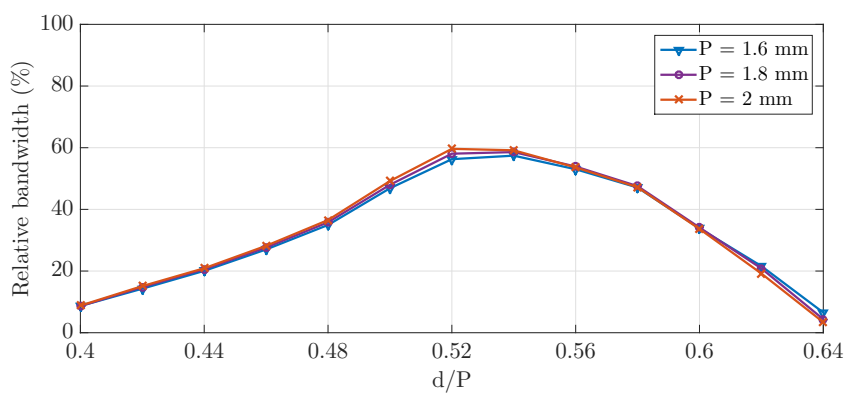

(a)

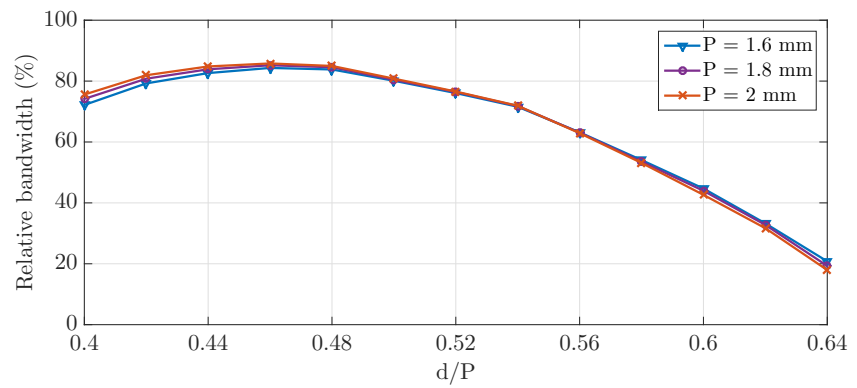

(b)

Fig. 5. Bandwidth of the stopband as a function of $d / P$ for a number of periods $\mathrm{P}$ with $\mathrm{h}=0.1 \mathrm{~mm}$ and $\mathrm{g}=0.01 \mathrm{~mm}$. (a) Unit cell with circular holes, (b) Unit cell with diamond-shaped holes.

less sensitivity to the gap size, and still works for larger gap sizes. Again this involves advantages related to the mechanical assembly, especially for complex waveguide components such as planar array antennas. Fig. 7 illustrates the effect of the thickness of the plates, which determines the depth of the holes (h). By increasing the depth of the holes the relative bandwidth of the stop-band increases, and it saturates when the depth is

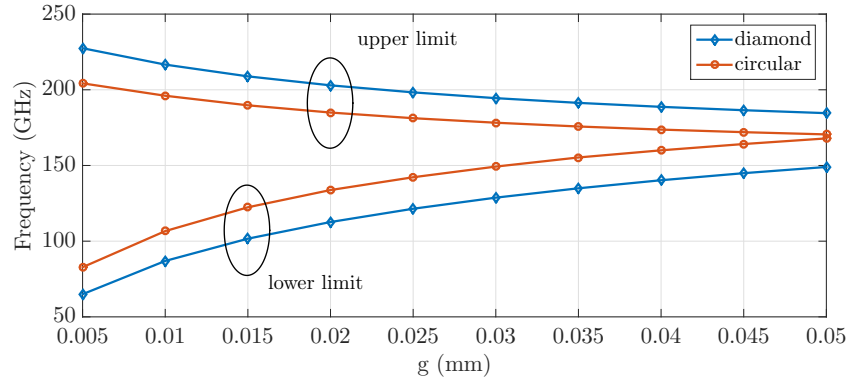

Fig. 6. Effect of gap $\mathrm{g}$ in the stopband for circular unit cell $(\mathrm{h}=0.1 \mathrm{~mm}, \mathrm{P}$ $=1.86 \mathrm{~mm}$, and $\mathrm{d}=1 \mathrm{~mm})$ and diamond-shaped unit cell $(\mathrm{h}=0.1 \mathrm{~mm}, \mathrm{P}=$ $1.86 \mathrm{~mm}$, and $\mathrm{r}=0.86 \mathrm{~mm}$ ).

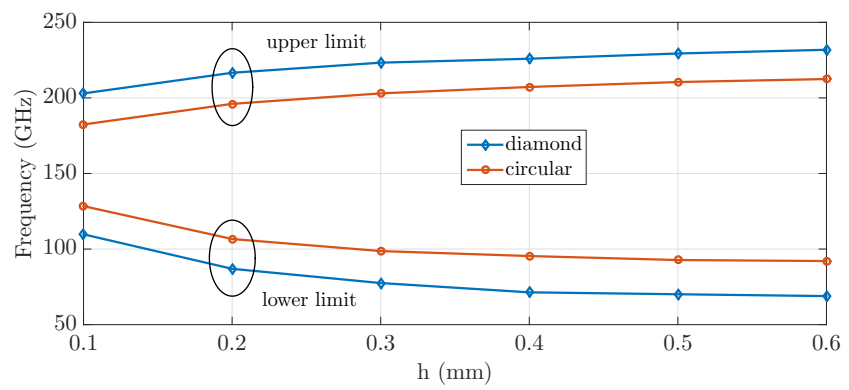

Fig. 7. Effect of depth of the holes $\mathrm{H}$ in the stopband for circular unit cell ( $\mathrm{g}=0.01 \mathrm{~mm}, \mathrm{P}=1.86 \mathrm{~mm}$, and $\mathrm{r}=1 \mathrm{~mm})$ and diamond-shaped unit cell $(\mathrm{g}=0.01 \mathrm{~mm}, \mathrm{P}=1.86 \mathrm{~mm}$, and $\mathrm{r}=0.86 \mathrm{~mm})$.

bigger than quarter of a wavelength at center frequency of the stop-band. In order to have one octave bandwidth the depth of the gap should be larger than $\lambda / 10$ at the center frequency of the stop-band. However, the unit cells provide a stop-band for thinner plates, with a smaller bandwidth.

\section{B. Straight MLW line with right-angle transitions}

A straight air-filled waveguide line with the proposed architecture is designed in order to investigate the performance of MLW. The designed line has two standard WR-6 inputs at the top to have a simpler interface with the measurement equipment. The geometry of the straight MLW transmission line is shown in Fig. 8. The waveguide line consists of five $200 \mu \mathrm{m}$ thick metal plates with $10 \mu \mathrm{m}$ gap between the layers. An elongated rectangular slot with a width of $1.65 \mathrm{~mm}$ is removed from the middle layers. After stacking the layers, a rectangular waveguide line with dimensions $1.65 \mathrm{~mm} \times 0.642 \mathrm{~mm}$ will be formed. It is worthy to mention that the height of the designed waveguide is slightly smaller than the standard WR-6 waveguide $(0.82 \mathrm{~mm})$ at D-band. Therefore, we expect slightly higher loss than the standard waveguide.

The length of the straight MLW line in Fig. 8 is $30 \mathrm{~mm}$, which corresponds to $15 \lambda_{0}$ at $150 \mathrm{GHz}$. The geometry of the transition is shown in Fig. 8(b). Simple steps are made by extending the layers into the waveguide channel to achieve a wideband input matching. Fig. 8(c) shows the middle layer (the layer \#3) with more details. We have removed a periodic rectangular slot along the waveguide channel in order to avoid the coupling of the wave into the gap between the layers. 


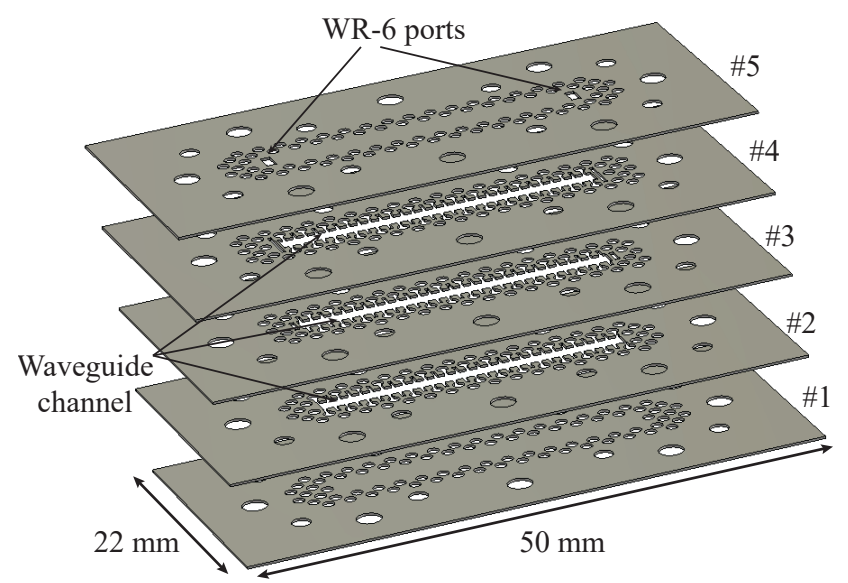

(a)

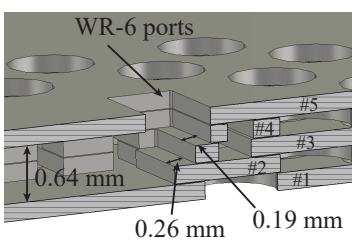

(c)

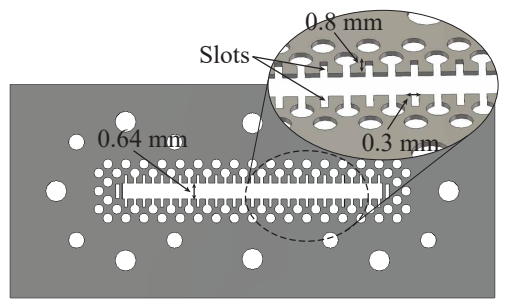

(d)
Fig. 8. The proposed multi-layer waveguide (MLW) transmission line configuration. (a) 3-D exploded view. (b) Cross-section view. (c) Top view of layer \#3.

Without the slots, the wave couples to the edges of the waveguide and propagates, which adds extra losses.

We have designed two straight MLW lines with the circular and diamond-shaped EBG hole unit cells. The simulated performance of the designed lines with two right-angle transitions are shown in Fig. 9. We have used CST Microwave Studio for all simulations. The simulated reflection coefficients are below $-16 \mathrm{~dB}$ in the whole desired frequency band (over 110$170 \mathrm{GHz}$ ), and below $-20 \mathrm{~dB}$ for the most of the band. The simulated transmission coefficient is better than $-0.5 \mathrm{~dB}$ for most of the band. Silver has been considered as constructing material in all simulations.

Fig. 10 shows the simulated amplitude of the E-field distribution within the different layers in two different frequencies (110 and $170 \mathrm{GHz}$ ). As expected, there is no field leakage within the layers, even though there is a small gap between the layers and they do not have galvanic contacts. As mentioned before, the EBG glide-symmetric holes prevent the wave propagation and unwanted modes within the stop-band.

\section{MLW line with double 90 degrees bend}

Any discontinuity such as bends and T-junctions can cause severe leakage. Therefore, to investigate the potential use of this technology for the design of more complex waveguide structures, we have designed a double $90^{\circ}$ bends waveguide line. The geometry of the proposed line is illustrated in Fig. 11. It has a similar configuration (number of layers, gap size, and right-angle transitions) as the straight line in Fig. 8. The total length of the line is $38 \mathrm{~mm}$.

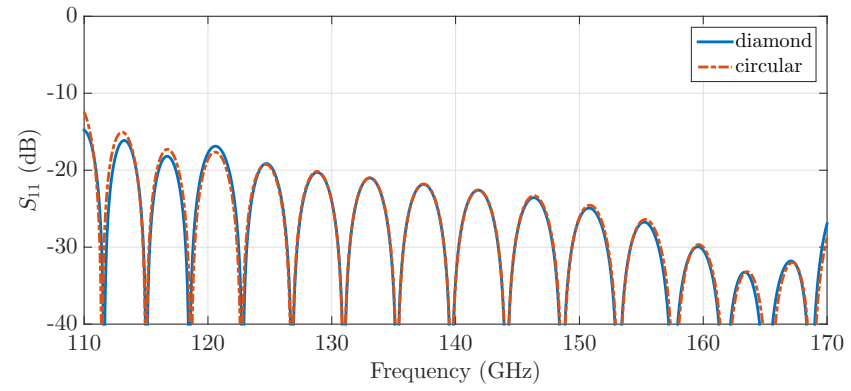

(a)

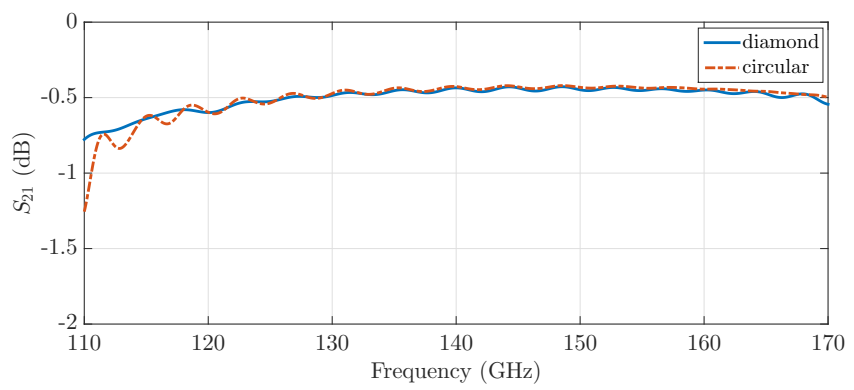

(b)

Fig. 9. Simulated performance of a $30 \mathrm{~mm}$ long straight MLW line with circular and diamond-shaped EBG unit cell. (a) Reflection coefficient. (b) Transmission coefficient.

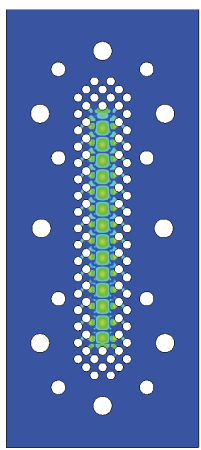

(a)

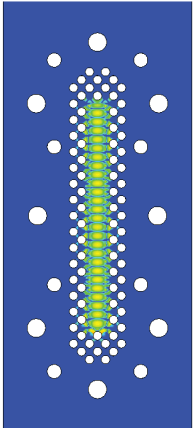

(a)

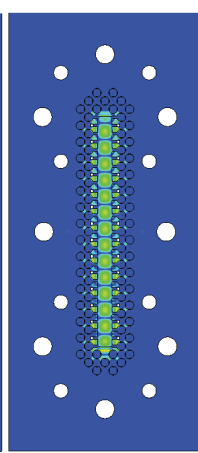

(b)

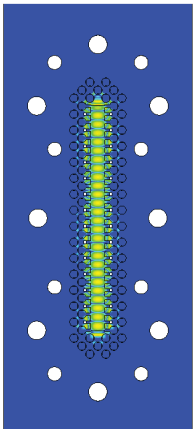

(b)

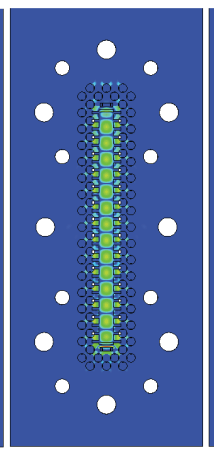

(c)

(a)

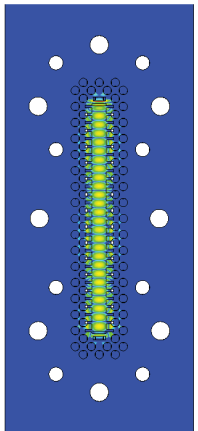

(c)

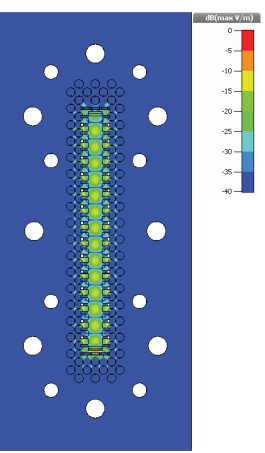

(d)

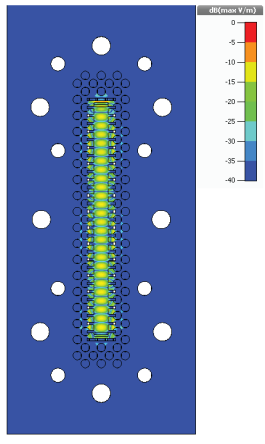

(d) (b)

Fig. 10. Simulated E-field distribution within the layers at (a) $110 \mathrm{GHz}$, and (b) $170 \mathrm{GHz}$ for a line with circular EBG unit cells (refers to Fig. 2).

The simulated S-parameters of the MLW double $90^{\circ}$ bends line are presented in Fig. 12. Two waveguide lines with the proposed circular and diamond-shaped holes EBG unit cells 


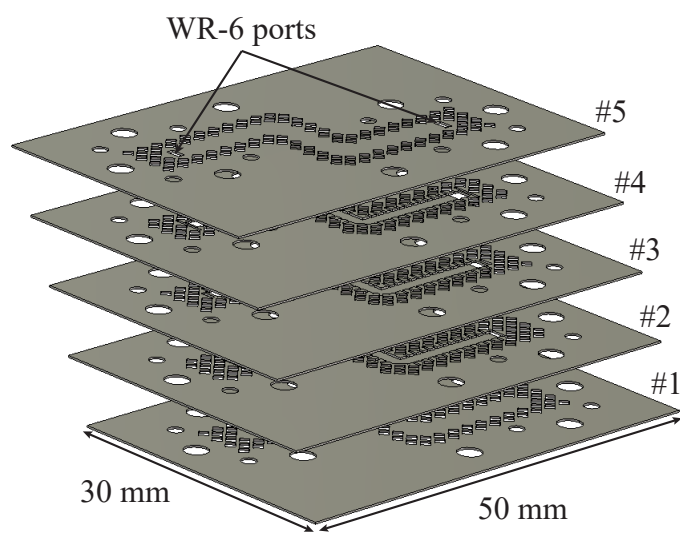

Fig. 11. Proposed multi-layer waveguide (MLW) transmission line with double $90^{\circ}$ bends.

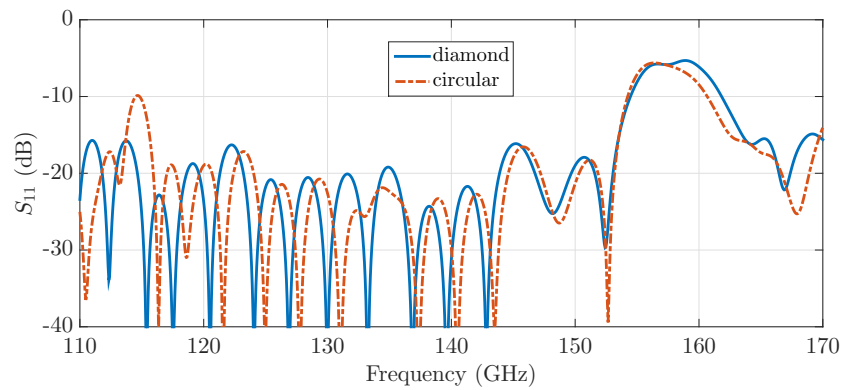

(a)

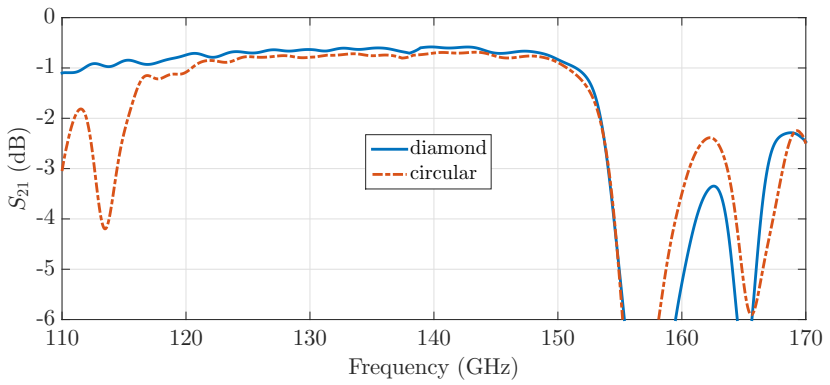

(b)

Fig. 12. Simulated performance of the MLW line with a double $90^{\circ}$ bends with circular and diamond-shaped EBG unit cell. (a) Reflection coefficient. (b) Transmission coefficient.

have been designed. Some resonances appear in the simulated results in Fig. 12, which degrade the performance and decrease the operating frequency bandwidth. The simulated reflection coefficients of the line with the diamond-shaped holes is below than $-18 \mathrm{~dB}$ over the frequency band $110-152 \mathrm{GHz}$, with the average $\left|S_{21}\right|$ value better than $-0.75 \mathrm{~dB}$ over the same frequency band. The line with the circular-shaped unit cell has similar performance, but there exists a small resonance at $115 \mathrm{GHz}$.

The simulated E-field distribution within the different layers at $140 \mathrm{GHz}$ is shown in Fig. 13. This figure verifies again that the EBG holes prevent field leakage and propagation of any wave in unwanted directions. We did an investigation to understand the reason of the unwanted resonance in Fig. 12. We found out that the location of the resonances are around

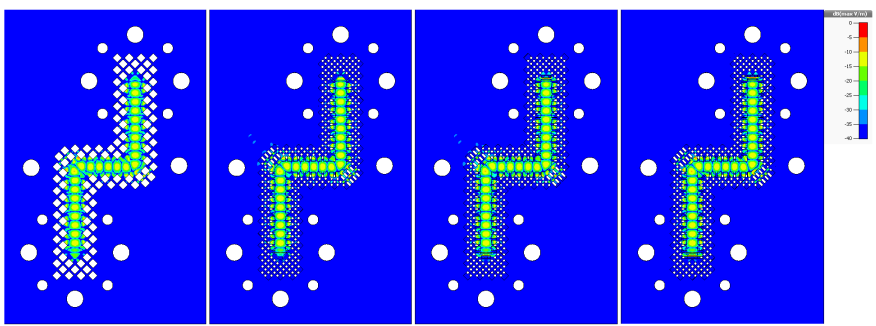

Fig. 13. Simulated E-field distribution within the layers at $140 \mathrm{GHz}$.

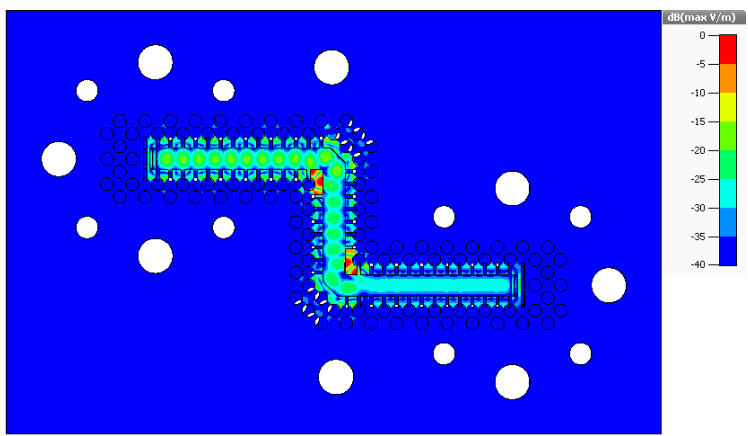

Fig. 14. Simulated E-field distribution within the layer number 4 at $155 \mathrm{GHz}$.

the corners of the bends. Due to the formation of parts at the designs with length of around one wavelength, a resonance accrues in the structure, as shown in Fig. 14. Fig. 14 shows the simulated time-averaged amplitude of the E-filed in the layer number 4 at $155 \mathrm{GHz}$. The red spots on the corners depicts the positions of the response. This problem can be solved by avoiding any metal sections with length of $\lambda$ and $\lambda / 2$, especially around the discontinuities.

\section{EXPERIMENTAL RESULTS AND DISCUSSION}

We have manufactured the presented MLW lines in Section II-A and B, as a proof-of-concept and performance validation. Fig. 15 shows the fabricated prototypes. The special pattern of each layer is made on a $200 \mu \mathrm{m}$ thick brass plate, by using metal chemical etching. The plates are silver plated in order to have lower conductive loss. The chemical etching provides a precise fabrication, which is scalable and suitable for mass production. Any arbitrary shape can be cut through thin metal plates with an aspect ratio of 1 . The manufacturing tolerance is approximately $\pm 20 \%$ of the metal thickness, which in our case becomes around $\pm 40 \mu \mathrm{m}$. Based on our experience, we have over-etching in most of the time, which makes the holes bigger than expected. Therefore, we have considered this effect and compensated the manufacturing tolerances in our design before fabrication. We decreased all the through holes dimensions $40 \mu \mathrm{m}$. By doing that, a maximum of $5 \mu \mathrm{m}$ error in the final fabricated parts has been achieved. The layers are simply assembled and glued together by using non-conductive epoxy glue.

The fabricated prototypes have been measured by using Keysight N5241A PNA-X and VDI WR-6 extenders, as shown in Fig. 16. The measured performance of the straight lines with circular and diamond-shaped holes is presented in Fig. 17. 

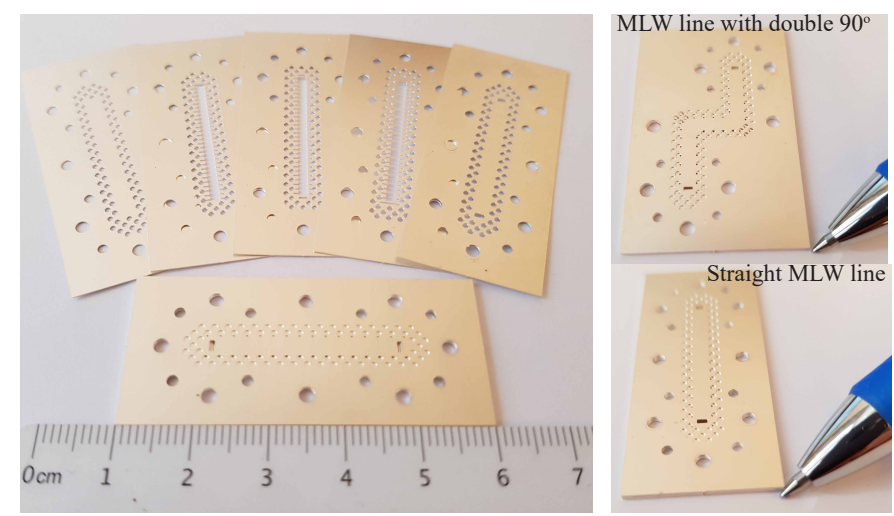

Fig. 15. Photograph of the fabricated MLW lines by using chemical metal etching.

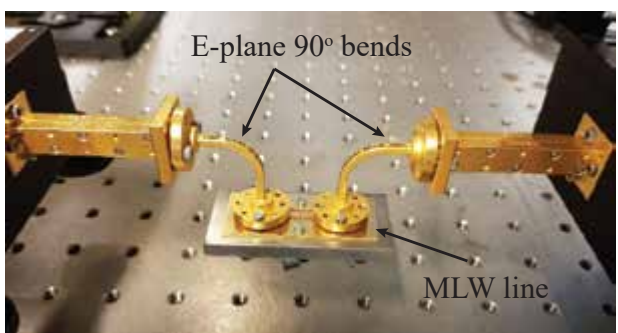

Fig. 16. Measurement setup.

The measured reflection coefficient is below $-18 \mathrm{~dB}$ over the frequency band $110-170 \mathrm{GHz}$, showing insertion loss values between 1.5-0.45 dB over the same frequency band. Both lines with different EBG unit cells show a similar performance. The measured reflection and transmission coefficients of the lines with two $90^{\circ}$ bends are presented in Fig. 18. The measured reflection coefficients are below $-18 \mathrm{~dB}$, with insertion loss between 1-0.65 dB, over the frequency band 110-150 GHz. As expected a resonance appears around $158 \mathrm{GHz}$, as discussed in Section II-B. The measured results in Fig. 17 and Fig. 18 are in very good agreement with the simulated results shown in Fig. 9 and Fig. 12, respectively. However, a slightly higher loss (around $0.1 \mathrm{~dB}$ ) and a small shift to higher frequencies are noticeable. These discrepancies are due to the existing higher conductive loss of the prototypes and fabrication and assembly tolerances.

\section{A. Comparison and discussion}

TABLE I shows a comparison between the proposed concept and several published works on different technologies. The commercial gold plated rectangular waveguide exhibits lower insertion loss than microstrip and SIW lines. The 3-D printed waveguides have comparable losses to the commercial one, after applying polishing techniques to maintain a low surface roughness. A very low insertion loss has been reported for the deep reactive ion etching (DRIE) silicon micromachined waveguide in [14], by achieving very low surface roughness. The average measured insertion loss per unit length of the proposed MLW line is around $0.02 \mathrm{~dB} / \mathrm{mm}$ at D-band. It should be remarked that the effect of the two right-angle

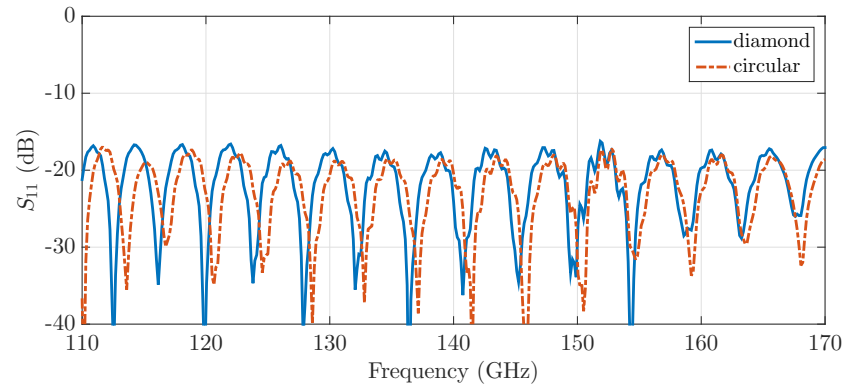

(a)

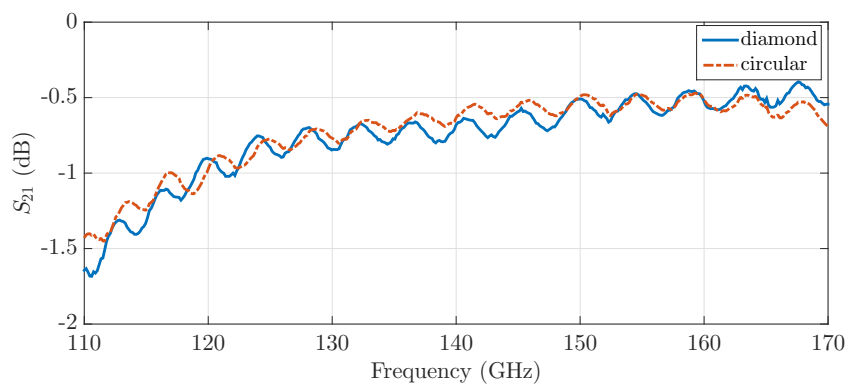

(b)

Fig. 17. Measured performance of the fabricated straight MLW lines at Dband. (a) Reflection coefficient. (b) Transmission coefficient.

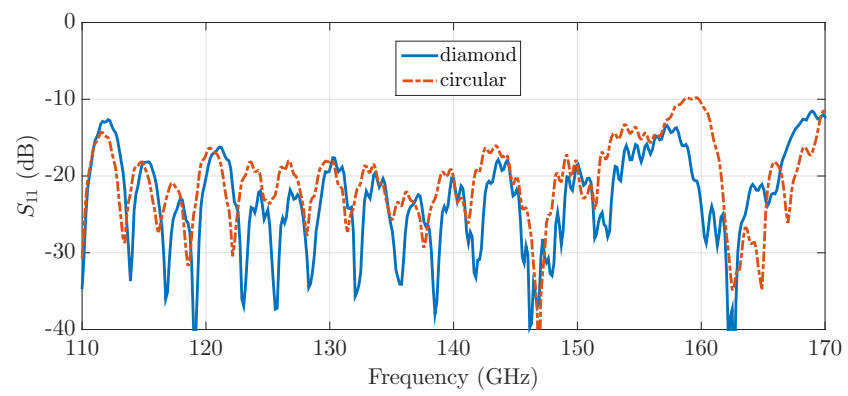

(a)

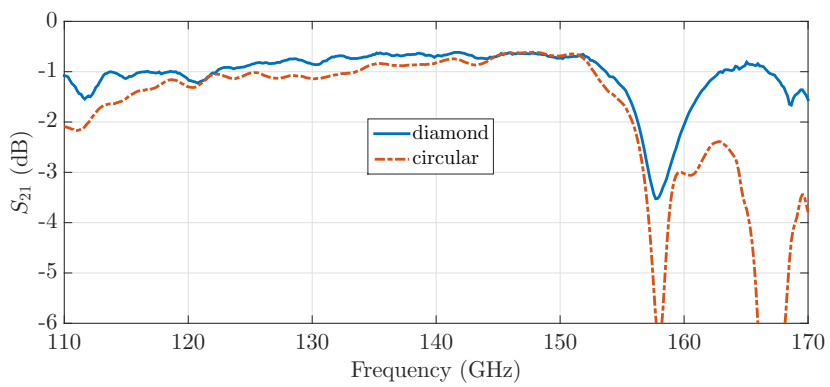

(b)

Fig. 18. Measured performance of the fabricated MLW line with double $90^{\circ}$ at D-band. (a) Reflection coefficient. (b) Transmission coefficient.

transitions are also included in the reported insertion loss. The presented transmission line shows a comparable performance to the commercial waveguide in terms of losses. Comparing with the dielectric based transmission lines, the proposed technology shows more than 10 times lower dissipative loss. Moreover, the proposed technology can be manufactured with very low cost techniques, by using mature fabrication methods such as metal laser-cutting and chemical etching. 
TABLE I

COMParison BetweEn STATE-OF-THE-ART WAVEguide TECHNOLOGIES

\begin{tabular}{|c|c|c|c|c|}
\hline Ref. & Technology & $\begin{array}{l}\text { Freq. } \\
(\mathrm{GHz})\end{array}$ & $\begin{array}{l}\text { Split- } \\
\text { block }\end{array}$ & $\begin{array}{c}\text { Loss } \\
(\mathrm{dB} / \mathrm{mm})\end{array}$ \\
\hline Commercial & - & $110-170$ & - & 0.012 \\
\hline [5] & Microstrip & $110-170$ & - & 0.33 \\
\hline [6] & SIW & $110-170$ & - & 0.35 \\
\hline [7] & $\mathrm{CNC}(\mathrm{Al})$ & $75-110$ & E-plane & 0.005 \\
\hline [13] & 3 -D printing 1 & $75-110$ & E-plane & 0.011 \\
\hline [8] & 3-D printing 2 & $220-325$ & r- & 0.012 \\
\hline [9] & 3-D printing 3 & $110-170$ & - & 0.019 \\
\hline [14] & micromachining 4 & $110-170$ & H-plane & 0.016 \\
\hline [11] & micromachining ${ }^{5}$ & $110-170$ & H-plane & 0.03 \\
\hline [12] & micro-molding & $75-110$ & H-plane & 0.03 \\
\hline This work & MLW & $110-170$ & H-plane & 0.02 \\
\hline
\end{tabular}

1 Stereolithography apparatus (SLA) with copper plating.

2 Stereolithography apparatus (SLA) with copper plating.

3 Selective laser melting (SLM) with $\mathrm{Cu}-155 \mathrm{n}$ powder

Deep reactive-ion etching (DRIE) in silicon with gold plating.

5 Multilayer silver plated SU-8.

\section{CONCLUSION}

We have presented a novel air-filled transmission line for millimeter-wave applications. In the proposed concept a waveguide is constructed by staking several thin unconnected metal plates. There is no requirement for having any electrical and galvanic contact between the layers. We have used EBG glidesymmetric holes to control the direction of propagation and eliminate field leakages. A straight line and a line with a double $90^{\circ}$ bends with two different EBG unit cells have been designed and fabricated. A high-precision and costeffective effective manufacturing method, i.e., metal chemical etching, is used to fabricate the prototypes. The measured performance of the fabricated prototypes were presented in order to verify the performance of the proposed concept. A very low-loss performance has been successfully achieved with average insertion loss of $0.02 \mathrm{~dB} / \mathrm{mm}$. The proposed unique architecture opens up an opportunity to provide lowloss and cost-effective millimeter-wave waveguide components and system packaging.

\section{REFERENCES}

[1] D. Lockie and D. Peck, "High-data-rate millimeter-wave radios," IEEE Microwave Magazine, vol. 10, no. 5, 2009.

[2] S. Carpenter, D. Nopchinda, M. Abbasi, Z. S. He, M. Bao, T. Eriksson, and $\mathrm{H}$. Zirath, "A D-band 48-Gbit/s 64-QAM/QPSK direct-conversion I/Q transceiver chipset," IEEE Transactions on Microwave Theory and Techniques, vol. 64, no. 4, pp. 1285-1296, 2016.

[3] D. Kim, J. Hirokawa, M. Ando, J. Takeuchi, and A. Hirata, "64×64element and $32 \times 32$-element slot array antennas using double-layer hollow-waveguide corporate-feed in the $120 \mathrm{GHz}$ band," IEEE Transactions on Antennas and Propagation, vol. 62, no. 3, pp. 1507-1512, 2014.

[4] K. Tekkouk, J. Hirokawa, K. Oogimoto, T. Nagatsuma, H. Seto, Y. Inoue, and M. Saito, "Corporate-feed slotted waveguide array antenna in the $350-\mathrm{GHz}$ band by silicon process," IEEE Transactions on Antennas and Propagation, vol. 65, no. 1, pp. 217-225, 2017.

[5] W. T. Khan, A. Ç. Ulusoy, and J. Papapolymerou, "D-band characterization of co-planar wave guide and microstrip transmission lines on liquid crystal polymer," in Electronic Components and Technology Conference (ECTC), 2013 IEEE 63rd. IEEE, 2013, pp. 2304-2309.
[6] S. Li, M. Yi, S. Pavlidis, H. Yu, M. Swaminathan, and J. Papapolymerou, "Investigation of surface roughness effects for d-band siw transmission lines on lcp substrate," in Radio and Wireless Symposium (RWS), 2017 IEEE. IEEE, 2017, pp. 121-124.

[7] I. Stil, A. Fontana, B. Lefranc, A. Navarrini, P. Serres, and K. Schuster, "Loss of WR10 waveguide across 70-116 GHz," in Proc. 22nd Int. Symp. Space Terahertz Technol, 2012, pp. 1-3.

[8] A. von Bieren, E. De Rijk, J.-P. Ansermet, and A. Macor, "Monolithic metal-coated plastic components for mm-wave applications," in Infrared, Millimeter, and Terahertz waves (IRMMW-THz), 2014 39th International Conference on. IEEE, 2014, pp. 1-2.

[9] B. Zhang and H. Zirath, "Metallic 3-D printed rectangular waveguides for millimeter-wave applications," IEEE Transactions on Components, Packaging and Manufacturing Technology, vol. 6, no. 5, pp. 796-804, 2016.

[10] X. Zhao, J. Bao, G. Shan, Y. Du, Y. Zheng, Y. Wen, and C. H. Shek, "D-band micromachined silicon rectangular waveguide filter," IEEE Microwave and Wireless Components Letters, vol. 22, no. 5, pp. 230232, 2012.

[11] D. Glynn, T. He, J. Powell, Y. Tian, X. Shang, and M. J. Lancaster, "Submillimetre rectangular waveguides based on SU-8 photoresist micromachining technology," in Microwave Conference (EuMC), 2016 46th European. IEEE, 2016, pp. 1346-1349.

[12] F. Sammoura, Y.-C. Su, Y. Cai, C.-Y. Chi, B. Elamaran, L. Lin, and J.-C. Chiao, "Plastic 95-ghz rectangular waveguides by micro molding technologies," Sensors and Actuators A: Physical, vol. 127, no. 2, pp. 270-275, 2006

[13] M. DAuria, W. J. Otter, J. Hazell, B. T. Gillatt, C. Long-Collins, N. M. Ridler, and S. Lucyszyn, "3-D printed metal-pipe rectangular waveguides," IEEE Transactions on Components, Packaging and Manufacturing Technology, vol. 5, no. 9, pp. 1339-1349, 2015.

[14] J. Campion, O. Glubokov, A. Gomez, and A. Krivovitca, "An ultra lowloss silicon-micromachined waveguide filter for D-band telecommunication applications," in Microwave Symposium (IMS), 2018 IEEE MTT-S International.

[15] P.-S. Kildal, "Artificially soft and hard surfaces in electromagnetics," Antennas and Propagation, IEEE Transactions on, vol. 38, no. 10, pp. $1537-1544,1990$.

[16] P.-S. Kildal, A. U. Zaman, E. Rajo-Iglesias, E. Alfonso, and A. ValeroNogueira, "Design and experimental verification of ridge gap waveguide in bed of nails for parallel-plate mode suppression," IET Microwaves, Antennas \& Propagation, vol. 5, no. 3, pp. 262-270, Feb. 2011.

[17] A. Vosoogh, P.-S. Kildal, and V. Vassilev, "Wideband and high-gain corporate-fed gap waveguide slot array antenna with ETSI class II radiation pattern in V-band," IEEE Transactions on Antennas and Propagation, 2016.

[18] H. Attia, M. S. Sorkherizi, and A. A. Kishk, "60 GHz slot antenna array based on ridge gap waveguide technology enhanced with dielectric superstrate," in 2015 9th European Conference on Antennas and Propagation (EuCAP). IEEE, 2015, pp. 1-4.

[19] A. Vosoogh and P.-S. Kildal, "V-band high efficiency corporate-fed $8 \times 8$ slot array antenna with etsi class ii radiation pattern based on gap technology," in Antennas and Propagation (APSURSI), 2016 IEEE International Symposium on. IEEE, 2016, pp. 803-804.

[20] J. Liu, A. Vosoogh, A. U. Zaman, and J. Yang, "Design and fabrication of a high-gain $60-\mathrm{GHz}$ cavity-backed slot antenna array fed by inverted microstrip gap waveguide," IEEE Transactions on Antennas and Propagation, vol. 65, no. 4, pp. 2117-2122, 2017.

[21] A. Vosoogh, A. A. Brazález, and P.-S. Kildal, "A V-band inverted microstrip gap waveguide end-coupled bandpass filter," IEEE Microwave and Wireless Components Letters, vol. 26, no. 4, pp. 261-263, Apr. 2016.

[22] A. Berenguer, M. Baquero-Escudero, D. Sanchez-Escuderos, B. Bernardo-Clemente, and V. E. Boria-Esbert, "Low insertion loss 61 ghz narrow-band filter implemented with groove gap waveguides," in Microwave Conference (EuMC), 2014 44th European. IEEE, 2014 pp. 191-194.

[23] M. Rezaee, A. U. Zaman, and P.-S. Kildal, "V-band groove gap waveguide diplexer," in Antennas and Propagation (EuCAP), 2015 9th European Conference on. IEEE, 2015, pp. 1-4.

[24] A. Vosoogh, M. S. Sorkherizi, A. U. Zaman, J. Yang, and A. A. Kishk, "An integrated Ka-band diplexer-antenna array module based on gap waveguide technology with simple mechanical assembly and no electrical contact requirements," IEEE Transactions on Microwave Theory and Techniques, vol. 66, no. 2, pp. 962-972, 2018.

[25] —_ "An E-band antenna-diplexer compact integrated solution based on gap waveguide technology," in Antennas and Propagation (ISAP), 2017 International Symposium on. IEEE, 2017, pp. 1-2. 
[26] M. Ebrahimpouri, O. Quevedo-Teruel, and E. Rajo-Iglesias, "Design guidelines for gap waveguide technology based on glide-symmetric holey structures," IEEE Microwave and Wireless Components Letters, vol. 27, no. 6, pp. 542-544, 2017.

[27] M. Ebrahimpouri, E. Rajo-Iglesias, Z. Sipus, and O. Quevedo-Teruel, "Cost-effective gap waveguide technology based on glide-symmetric holey EBG structures," IEEE Transactions on Microwave Theory and Techniques, vol. 66, no. 2, pp. 927-934, 2018.

[28] E. Rajo-Iglesias, M. Ebrahimpouri, and O. Quevedo-Teruel, "Wideband phase shifter in groove gap waveguide technology implemented with glide-symmetric holey EBG," IEEE Microwave and Wireless Components Letters, vol. 28, no. 6, pp. 476-478, 2018.

[29] M. Ebrahimpouri, A. A. Brazalez, L. Manholm, and O. Quevedo-Teruel, "Using glide-symmetric holes to reduce leakage between waveguide flanges," IEEE Microwave and Wireless Components Letters, vol. 28, no. 6, pp. 473-475, 2018. 